\title{
Diabetes insípida nefrogénica: Presentación de tres casos. Breve revisión del tema
}

\author{
Dras, P. Maldonado S.,* \$. Pino K.,** C. Noreru V.***
}

\author{
ABSTRACT \\ On present 3 cases of Nephrogerie Diabetes lnsipidus controled at the Department on Infant Nephrology - Hospi- \\ tal Sian Juan de Dios. \\ A review is made of the disease in relation ta the cases.
}

La diabetes insipida nefrogénica (DINi) es un desurden hereditario del riñon, caracterizado por la insensibilidad a la acción antidiurética de la vasopresina u hormona antidiurétics $(\mathrm{ADH})^{11}$, en la cual se produce poliuria abundante, hipostenuria, con usmolaridad y densidad baja de orina.

Esta enfermedad llamó la atención hace 34 años cuando Waring $y$ colaboradores deseribieron seis casos en 1945. (2) Posteriormente William y Henry (3), notaron la incapacidad de estos pacientes para cencentrar orina aun después de grandes dosis de hormona antidiurética, suficientes como para provocar efectos colaterales, con lo cual propusieron la teoria de la reiractariedad a la acción de la $\mathrm{ADH}$ en la DIN.

Existe también otro tipo de DIN no hereditaria, inducida por drogas, tales como la demeclocyclina. que produce un daño transitorio, reversible. dependiente de la dusis' 1 ' y últimamente se han apurtado más casos de DIN inducida por sales de litio. generalmente reversible $y$ dependiente de la dosis. $(5$ ) El presente trabajo tiene por objeto presentar tres casns clínicos de DIN controlados en el Departamento de Nefrología Infantil del Hospital San Juan de Dios y hacer una breve revision del tema.

Clasificación. Existen dos tipos fundamentales de diabetes insípida: las pitresino sensible, producidas por ausencia o disminución en la producción de

\footnotetext{
* Servicio Pediatria Hospital San Juan de Dios, Medico Pedialra Sanidal F.A.Ch.

* Becada Srrviciu do Pediarria Hospital San Juan de Dios.

*** Dires 1ora Deplar. de Pediatría, Facultad de Medicina Occiden-

te. Cniverajad de Chile.
}

ADH. y las pitresino resistentes, en que existe producción de ADH. pero hay falla en la respuesta del efector renal.

\section{CLASIFICACION DE LOS SINDROMES POLIURICOS'6!}

I ADH. Suprimido o ausente

1. ADH. Secreción suprimida. Polidipsia primaria.

2. ADH. Secreción disminuida o ausente:

Diabetes insipida central.

II. ADH. Secreción adecuada

1. Disminución en la generación y mantención de la gradiente osmótica.

a.- Hipokalemia

b.- Hipercalcemia

c.- Desnutrición

d.- Diuresis osmótica (glucosa, urea, sal, manitol, insuficiencia renal crónica)

e.- Diuréticos "de asa"

f.- Desúrdenes de sickle cell

g.- Síndrome de hiperviscosidad

h.- Nefropatías lúbulo-intersticiales (pielonefritis crónica, nefropatía por analgésicos, nefritis por radiación, enfermedad cística medular, hidronefrosis, infecciones urinarias no ubstructivas, síndrome de Sjögren, riñón de trasplante, amiloidosis).

2. Utilización disminuida de la gradiente osmítica.

a) Respuesta disminuida de los túbulos colectores a la ADH 
Diabetes insipita nefrogénira

Drugas tcarbonato de litio. de mechoriclina, colchisina)

Hipercalcemia

Amiluidusis

b) Inadecuada velocidad de flujo urinario Diuresis usmótica

Diuresis pustobstructiva

Frecuencia. No hemos encontrado publicaciones con datos estadísticos referentes a la frecuenciu de la enfermedad. En C.S.A. se supone que la enfermedad llegi al pais en el siglo XVIII, con inmigrantes provenientes de Escocia. ${ }^{17}$

Patogenia. Los estudios genéticos efectuados hasta ahora por múltiples autores, favorecen la hiputesis de que esta enfermedad se transmite por herencia ligada al cromosoma $\mathrm{X}$, observándose que las madres transmiten la enfermedad a los hijos varones, pero también se han descrito casos de mujeres afectadas por la enfermedad. Carter y Simpkiss ${ }^{|8|}$, demostraron defectos de concentración variables en mujeres heterocigotas, después de una prueba de privación de agua. En general, se acepta que la enfermedad es más suave en las mujeres que en los varones. Otro hecho clinico observado es que la enfermedad se asocia a retardo mental y del crecimiento. ${ }^{(9)}$

Fisiopatología. La hormona antidiurética se forma en el núcleo supraóptico del hipotálamo. De allí desciende por los axones del tálio hipotálamo-hipofisiario y es almacenada en el lóbulo posterior, en estrecho contacto con los capilares sanguíneos. Es un octapéptido de P.M.: 1.000 aproximadamente. Según estudios realizados circula libremente en forma de octapéptido y puede atravesar membranas capilares hacia el espacio extravascular. No se ha demostrado que la ADH circule unida a una proteína.

La ADH se puede medir en el plasma sólo por métodos biológicos. Heller y Stule, encontraron una actividad antidiurética promedio de $1.67 \mathrm{C} / \mathrm{ml}$. en el plasma en sujetos normalmente hidratados. Estos mismos autores demostraron que la actividad antidiurética del plasma aparece a los cuatro meses de vida alcanzando hacia los 14 años el valor de un adulto.

Al parecer se inactiva parcialmente en higado y riñón, pero no eslá clara la participación de otros tejidos en su metabolismo.

La ADH tiene varias acciones biológicas:

1) Estimula la contracción del músculo liso vasurular, aparentemente sensibilizindolu a la accoin de las catecolaminas.

2) Promueve la reabsorción de agud a nivel del rubuto renal.

Está comprobado que en la DIN la ADH está presente en la circulación, pero es el nefrón el que no puede responder. En los rinimes, la respuesta normal a la ADH es esencialmente un aumentı en la permeabilidad al agua en los túbulos colectores. Esta respuesta sería medida por el adenosín monofosfato cíclico (c AMP).1111, 12, 13, 14, 15. 16. Se ha comprobado que la adición de $\mathrm{ADH}$ al hornogenizado de riñón o fracción de membrana. produce un aumento de la producción de c AMP in vitro.113. 15) También se ha observado que la excrecicin urinsria de c AMP puede aumentar en respuesta a la $\mathrm{ADH}$, pero otros autores no han logrado demostrar un cambio significativo. Se acepta que se une a un receptor especifico. activando la adenilciclasa, la cual cataliza la conversión de ATP en $c$ AMP. Este último actuando como mediador intracelular para la $\mathrm{ADH}\left(14\right.$. l $^{2}$ ) es degradado a 5' AMP por la enzima c AMP fosfodiesterasa, la cual no es influenciada por la ADH. Esta enzima es inhibida in vitro por la teofilina, aminofilina y clorpropamida. $\{1\}, 13,191$

Los receptores de la membrana citoplasmática asociados a la adenilciclasa, se ubican en la superficie externa y la adenilciclasa en el inferior $\left(2^{0}\right)$ La unión hormona receptor es una unión estereospecífica y el número de sitios de unión es limitado.

El sistema receptor y adenilciclasa estáı asociados por algún mecanismo catalítico desconocido hasta ahora pese a los estudjos realizados.

En preparaciones celulares el aumento de la dosis de ADH en la estimulación de la adenilciclasa conduce a una curva clásica en $S$ itáliça, lo cual confirma la afinidad de la hormona por este sistema.!20

Los análisis quiméticos de los movimientos del agua a través de la membrana epitelial, sugieren que un aumento en el paso de flujo a través de la gradiente hidrosmótica producida por la ADH o el c AMP es debida a un aumento en el tamaño o número de los poros de la membrana.' 13, 21! También la acción del c AMP se acompaña de cambios en las propiedades mecánicas de la membrana luminal. 1221 Se han invocado yarias teorias de cómo el c AMP induciria estos cambios estructurales y funcio nales'20!. pero al respecto los mecanismos más intrínsecos son desconocidos. 
Evidencias indirectas apoyan la idea de que la proteinquinasa activada por el c AMP podria fosfosilar las proteínas de la membrana citoplasmática y ésta podría ser la base para un cambio en la permeabilidad al agua.

Además, estudios hechos en vejiga de sapo demuestran que la ruptura del sistema nic rotubular de las cćlulas que se efectúa por medio de ciertas drogas, inhibe el efecto hidrosmótico de la ADH y del c AMP. Taylor y colaboradores postulan que se necesitaría integridad del sistema microtubular para que el mediador : AMP produjera los cambios en la permeabilidad de la membrana citoplasmática. Esto mismo parece aplicarse a riñones de mamíferos. 231

Sin embargo, los últimos años, diferentes trabajos clínicos parecen demostrar que la DIN sería un desorden heterogéneo, ya que en algunos casos se ha observado que la administración de $\mathrm{ADH}$ produce aumento de la excreción urinaria de c AMP. lo cual indicaría que la falla estaría en estos casos, más allá de la producción de c AMP por la estimulación del sistema adenilciclasa por la ADH. Esto haría discutible el valor de la determinación de c AMP urinario en el diagnństico diferencial de las nefropatías debidas a fallas de la concentración. . $^{(24)}$

Zimmerman y Green publicaron en 1975 un caso de DIN en una niña, en la cual la exc:reción urinaria de c AMP aumentaba en un $89 \%$ en respuesta a la ADH. Ellos proponen la existencia de dos tipos de DIN: la tipo I, clásica, ligada al cromosoma $X$, sin aumento del c AMP en respuesta a la ADH. En la tipo II, el c AMP urinario aumenta con la administración del $A D H$ y no estaría ligada al cromosoma X.1261

Caso .N." 1. MACR Sexwo: masculino. F.N.: 23 Enero 68. P.N. 3.500 grs.

Parti: N.

Consulea mil atro hospital, porgue desde los $4 \mathrm{~m}$. de edarl la marle nota irngestude liquidos de más o menos 3 li. al dia y poliuria, falia de ascenso pondoestatural a pesar del buen aporte y buena ingestu de aljmentos. Con la suspension del agua presentaba sertuedad de la buca. desesperación, sensación febril que cede con piramidén. Vírnitos postorandiales ocusiunales.

\section{ANTECEDENTES FAMILIARE5. Gráfico 1. \\ Examen fisicor de ingreso: Tabla J. \\ Exámenes de laboralorio: Tabla 2.}

Con estos antecedentes se dïagrustici DIN. Se iniciti tratamientu con régitmen sin sal. HCT, KCL, con lo cual disminuye la ingesta dr agua y la diuresis se reduce en un $50 \%$ con densivad 1.004. La hipernatremia se corrıgio.

Se controla irregularmente an Hospital San Juan de Dios. desde bace 4 años. Se comprueba retardo psicorantor y ponduestatural. A tratamiento inicial se agregó furosemida con bucnos resullados mientras se mantiene la terapib. Su controi es espordidico por problemas familiares, siempre es necesario darle agua en la noche, pues de lo contrario se deshidrata. Su ansia de agua es Ian importunte yue en una oportunidad se quemá la boca y el carifago pur tralar de tomar agua desde una tetera hirviendo.

\section{ARBOL GENEALOGICO Fam. L. B.}

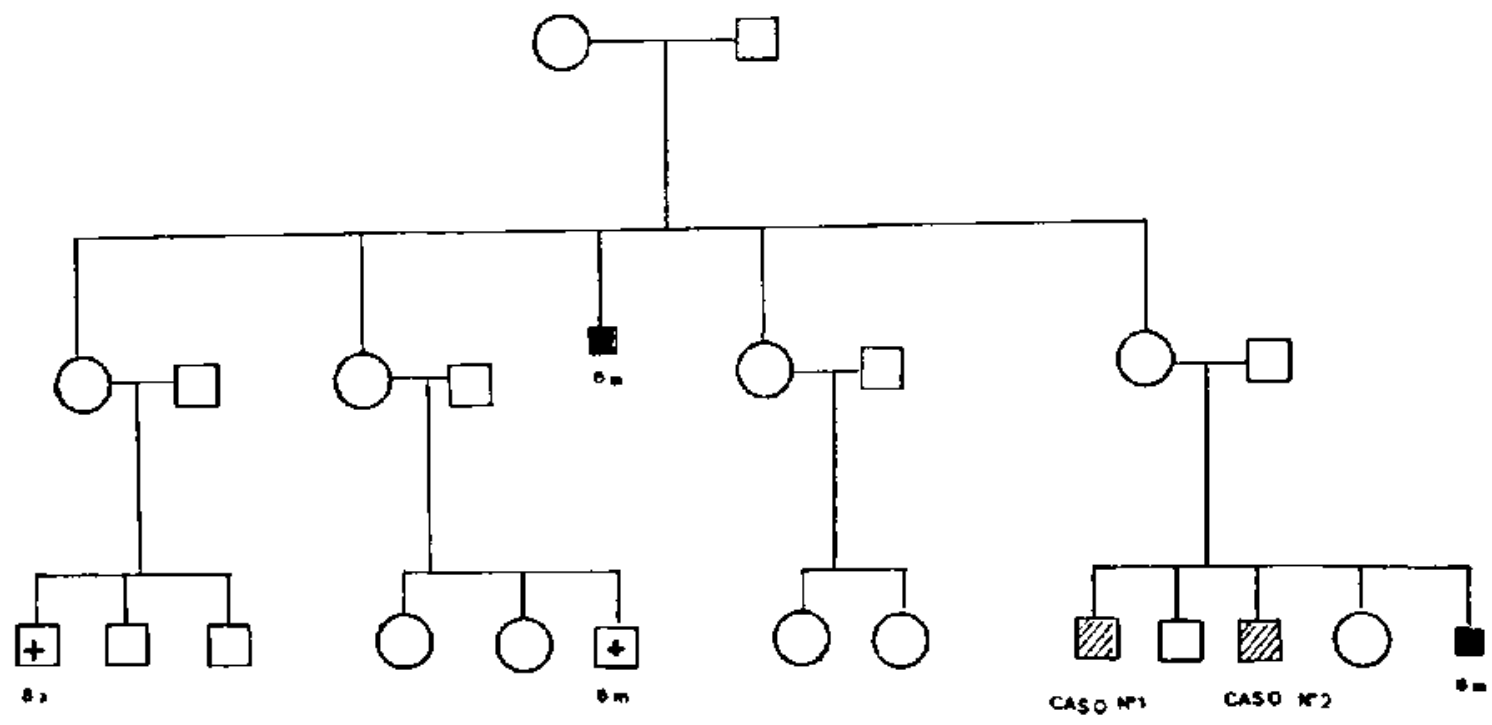


Caso N." 2. PLB Sexo: masculino. F.N. J4 Mayu 60, P.. V. 2, 7t)0 grs. Parto: X.

Paciente que a la edad de $2 \mathrm{~m}$, fue hospicalizado por cuadro diarreicu aqudo y. Bn. que se recuperaron con tratumiento habituat. Duranle la evolución presentó deshidratación y alzas térmicas que alcanzaron los $39 \mathrm{c}$, vómitos repetidos, anemia, y se pensó en cuadro infeccinso subyutente. Se descarto TBC., y/o infección urinaria y sepeis. No se completi el estudio por huelga de persnnal. Desde entunces, la madre nota que el njôno ingiere grandes cantidades de azua, incluso en la noche. Sin 1umarla se deseeperaba y se deshidrata rúpidumente. A los 10 años de edad. ingresú en otro hospital para ser estudiado por haberse encuntrado a un hermano suyo, portador de DIN (Casn N." l). Presenta diure sis de miás de $\hat{i}$ mil cc. en $\mathbf{2 4}$ huras y relardo psiçuico impurtanle. Exámen fisico: Tabja 1.

Exámenes de laboraturio: Tubla 2.

\begin{tabular}{|c|c|c|c|c|c|c|}
\hline $\mathrm{N}^{*}$ DE CASO & \multicolumn{2}{|c|}{1} & \multicolumn{2}{|c|}{ z } & \multicolumn{2}{|c|}{3} \\
\hline$\$ E \times 0$ & \multicolumn{2}{|r|}{$\sigma^{2}$} & \multicolumn{2}{|c|}{ 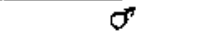 } & \multicolumn{2}{|c|}{$\bar{\zeta}$} \\
\hline & INGRESO & ACTVAL & IHGRESO & ACTUAL & INGRESO & ACTYAL \\
\hline$E D A D$ & $203 \mathrm{~m}$ & 110 & oa & $18 \mathrm{a}$ & $2 \mathrm{a}$ & Sa \\
\hline FESOY & $6.2 \mathrm{~kg}$ & $25 \mathrm{~kg}$ & $22 * v$ & $58 \mathrm{~kg}$ & as $\mathrm{kg}$ & $16 K_{0}$ \\
\hline $\begin{array}{l}\text { PERCENTIL } \\
\text { \{para odad\} }\end{array}$ & Bopo 3 & Bap p 3 & 801003 & $D 10$ & $E_{10} D_{3} 3$ & g 10 \\
\hline TAELA Y & $69 \mathrm{~cm}$ & $120 \mathrm{~cm}$ & $1 ! 3 \mathrm{~cm}$ & $156 \mathrm{~cm}$ & $25 \mathrm{~cm}$ & $107 \mathrm{~cm}$ \\
\hline $\begin{array}{l}\text { PERC ENIIIL } \\
\text { t paro odad I }\end{array}$ & Bapa o 3 & 801003 & 84003 & Sopo 3 & 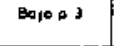 & P 25 \\
\hline$P A$ & N & $w$ & n & $N$ & $\mathrm{~N}$ & N \\
\hline OLDED YESICAL & $\cdots$ & * & $\cdots$ & + & - & - \\
\hline EX. NEUROLOGICO & $\begin{array}{l}\text { Retordo } \\
\text { psicomber }\end{array}$ & id & $\begin{array}{l}\text { Raiardo } \\
\text { ssicomoter }\end{array}$ & id & Leveretor & Nermal \\
\hline $\begin{array}{l}\text { INGESTA DE AGUA } \\
\text { SIX TR. }\end{array}$ & 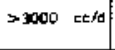 & 3,5000 ettd & $>7000 \leqslant 10$ & $>0500 \times d d$ & $22500 \mathrm{xctd}$ & $3 \times 4000 \times A$ \\
\hline $\begin{array}{c}\text { DINFESLS DIAFIA } \\
\text { SIM IR }\end{array}$ & $>2 \cos c t d$ & $>10000 \times 0</ d$ & $=7000 \mathrm{t} 5 \mathrm{~s}$ & $=5100 \Leftrightarrow d$ & $>2000 \mathrm{ct} / \mathrm{d}$ & $>4000 \mathrm{c} / \mathrm{d}$ \\
\hline DENSIDAD URINARIR & 1003 & $100 \mid$ & 1003 & 1003 & 1000 & 1002 \\
\hline
\end{tabular}

EXAMENES DE LABORATORIO

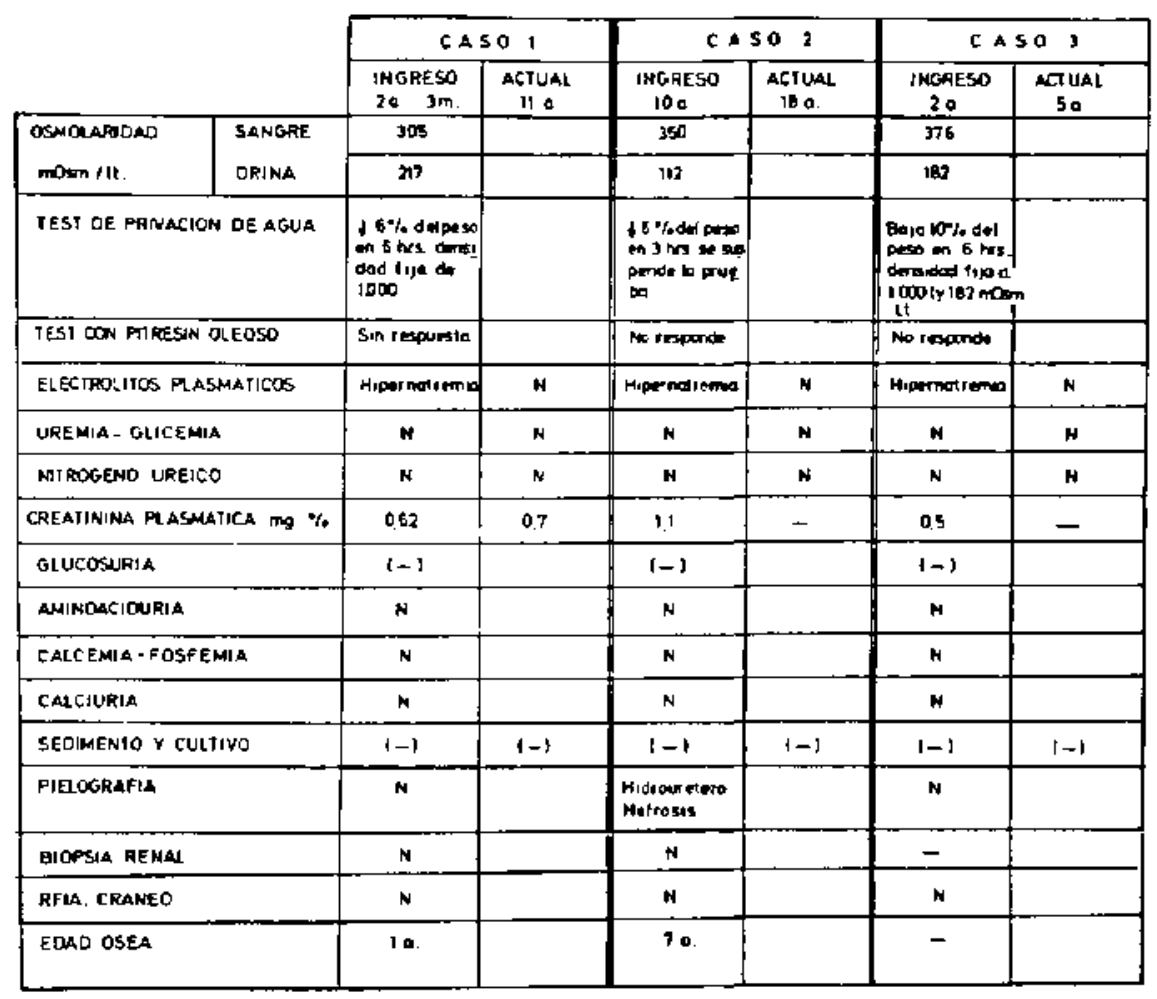


Se diagnosticó DLV. Tralado eun turusemide se ubservó reducción de la diuresis a 2.300 ce al dia. Dejo el contrul en 1972. Desde 1973, empiexa a cuntrolarse nuevamente en furova esporadica: actualmente liene 18 añas y cursa ontava básice en Escuela Namsturna.

Caso N." 3. JC.V. Sexo: masculino, F.N. 8 May, 1973. P.N. 2.800 grs.

Parto: $\mathrm{N}$.

Fup huspitalizado a los 2,6 y 8 meses de vida por rovaltos de lipertermia en los cuales se suspectui infecer ión no bahiéndose compribado etiologia en ninguna de esas uportunidades. Durante esos mesers la inadre nola que el nino ingiese grandes cantidades de agua. Despurs de los 3 meses de edad presenta vionitos postprandiales precuces $y$ dolor atodominal orasionales, En todas estas horpitalizacinnes se observa leve retraso pondoestatural y psicomotur. A lass 2 añus se esntrolaba por desuurriciniu leve. Lin romilidu para su estudio a la colad de 2 añis al Servicio de Pedintrias de nuestro Haspital.

ANTECEDENTES FAMHLIARES. FTRÁficu 2.

Examen fipica: Tabla $\mathbf{l}$.

Fxámencs de laboratorio: Talbla 2.

Con los antecedentes previos se diagnustion DIX. Se

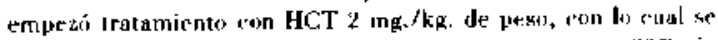
aprecia disminucisin de la ingesta de agua en más a menrs $30 \%$. Sie ba contrulada regulacmente apreciándone buen destarrollo psicurnotor. Actualmente rectibe HCT $5 \mathrm{mg} . / \mathrm{kg}$; rigimen sin sal y KC:L jarabe.

\section{DIABETES INSIPIDA NEFROGENICA}

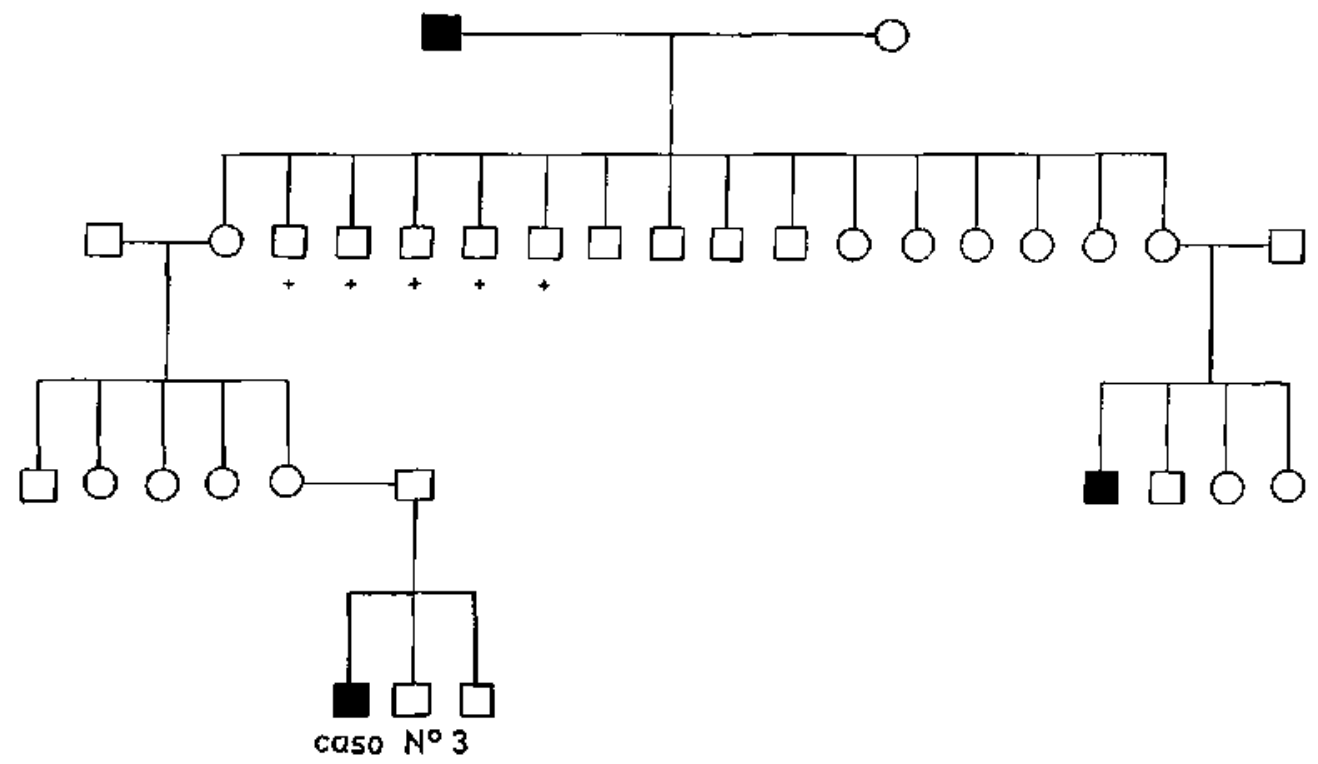

\section{COMENTARIO}

Lo más llamativo de esta enfermedad es la intensa avidez por el agua y las espectaculares poliurias que. presentan estos niños.

Por lo tanio, deberíamos plantear el diagnóstico de DIN frente a un lactante con poliuria, avidez por el agua y tendencia a la deshidration, ya que el diagnóstico precoz disminuye las secuelas propias de esta enfermedad tales como el déficit intelectual y pondoestatural, además de otras complicaciones próximas como las deshidrataciones graves y los peligros de la hipernatremia. Frente a un lactante deshidratado y con poliuria deben distinguirse las situaciones que llevan a la producción de diuresis excesiva en esas condiciones. Debe plantearse el diagnóstico diferencial entre diabetes insípida idior pática, diabetes mellitus, pielonefritis crónica con uropatía malformativa o displasia renal, nefritis perdedoras de sal y desnutrición con hipokalemia.

La mayoría de estos cuadros se caracteriza por presentar una alteración en la capacidad de concentración renal, que se manifiesta con poliuria, pero la diuresis no alcanza las cifras excesivas de la diabeles insípida y de la DIN. Para diferenciar ambos cuadros el test de Pitresin es fundamental. puesto que no habrá respuesta en la DIN.

Las cifras de densidad urinaria son orientadoras inicialmente para plantear los diagnósticos diferenciales: en la DIN nunca la densidad sube de 1.005 (excepcionalmente, en condiciones de extrema deshidratación puede llegar a 1.008): en la diabeles 
mellitus la orina es isotónica: en la pielonefritis crónica hay isostenuria hasta las etapas finales de la enfermedad, con densidad de alrededor de $1.010 \mathrm{y}$ con valores de diuresis más bajos; en el lactante desnutrido y con hipokalemia, factores ambos que alteran la capacidad de concentración renal, las diuresis no son tan importantes y las cifras de densidad urinaria son más altas. Es útil la determinación de electrolitos plasmáticos: en cuanto al sodis, las nefritis perdedoras de sal se caracterizan por hiponatremia. al contrario de la DIN. En cuanto al potasio, cabe destacar el síndrome de Barter, con severa hipokalemia, cifras altas de renina plasmática y de aldostermia comprobándose en la biopsia renal hipertrofia del aparato yuxtaglomerular.

En el niño mayor debe plantearse el diagnóstico diferencial con la polidipsia psicogénica, y es aquí dónde la prueba de deprivación de agua adquiere su máximo valor.

Nuestrns casos en su totalidad se ajustan a las caracteristicas de la DIN. Los tres niños presentaron episodios febriles durante el primer año de vida, avidez por el agua con poliuria importante. Dos de ellos con retardo psicomotor y pondoestatural importante, que estarian derivados de los episodios de hipernatremia en la etapa prediagnóstica y en la avidez por líquidos hipocalóricos en vez de la alimentación sólida. Además en estos casos hay tratamientos mal llevados por inconstancia de los pacientes. Todos han tenido grandes poliurias, con hipostenuria acentuada y baja osmolaridad en contraste con pruebas de función glomerular normal e indemnidad del parenquima renal. Las pruebas de función tubular fueron normales a excepción de la incapacidad de concentrar la orina, bo cual no fue modificado por la ADH en el test de pitresin oleoso.

En cuanto a la presencia de hidrourétero-nefrosis (Caso 2), esta es una complicación mecánica como consecuencia de las grandes poliurias. 2 ?"

Aunque parezca paradojal, el tratamiento de estos enfermos se efectúa con diuréticos. La efectividad de las tiazidas y de otros diuréticos eliminadores de sal, en dísminuir la poliuria, parece depender de una contracción del LEC con aumento de la reabsorción proximal del filtrado glomerular, disminuyendo la entrega de agua al túbulo distal. Esto lleva a una disminución del clearence de agua libre $y$ aumento del clearence osmolar.128, 29)

Por ello es importante la restricción de sal en la dieta, ya que se observa una disminución de la diuresis independiente del efecto de las tiazidas. Esto lo observamos claramente en la evolueión del caso N." 3 , en que al ingerir una dieta de 4 grs. de sal se produjo un aumento de la poliuria a pesar del tratamiento tiazidico. Al suspender la sal, la diuresis volvii a cifras hahituales.

Actualmente, se plantea el Iratamiento de diuréticos ascriados a inhibidores de las protaglandinas. puesto que se ha observado empírieamente mayor disminución de la diuresis con el tratamiento de hidroclorotiazida $5 \mathrm{mg} / \mathbf{k g}$ más aspirina $80 \mathrm{mg} \% \mathbf{k g}$ 1 d. ${ }^{134}$ El mecanismo por el cual se observa este efecto no está aclarado en absoluto y merece mayores estudios físiopatológicos.

\section{RESEMEN}

Se presentan 3 casos de Diabetes Insípida Nefrogénica, controlados en el Departamento de Nefrologia Infantil del Hospital San Juan de Dios.

A propósito de los casos se hace una breve revisión de la enfermedad.

\section{REFEREINCIAS}

${ }^{1}$ N. F. Jones; M. A. Barraclough ef als. "Nephnogenic Diabetes Insipidus. Effecto of '3' 5 ' Cyclic - Adenosin Monophonphate." Areb. of Dis, in Childhood 1972, 47, 794.

2 Waring, Kajd and Tappan, 1445 . "Congenital Defects of Waler Metabolism." An. J. Dis. Child. 69: 323, 1945.

3 William, R. H., and Henry. "Nephrogenic Diabetes Insipidua: Trensmitted by Fernalls and Appearing During the Infancy in Males." Am. Int. Med. 27: 64-95, 1947.

4 Irwing, Singer, M. D., F.A.C. P. and Donald Rotemberg, $M$. D. Philadelphia, Pennsylvanis. "Demeclocycline. Induced Nephrogenic Diabetes Insipidus." Annals of Interual Medicine, 79: 679-683, 1973.

5 Norman, Simon et als. "Pereistent Nephrogenic Diabetes Insipidus After Lithium Carbonate." (Letter) Annals of Interng] Medicine 86 (4): 446-447, Apr. 1977.

"Ulrich, F.; Michael, M. D. "Poliuric Syndrome." Arix. Med. 35 (4): 264-273, Apr. 1978.

${ }^{7}$ Hans, H.; Bode, M. D., et als. "Nephrogenic Diabetes Ineipides in North America." The Hopewell Hypothesis. The New Engl. J. of Med. Apr. 3, 1969.

Barter and Simpkiss, $M$. " Carrier's State' in Nephrogenic Diabetes Insipidue." Lancet 2: 1069, 1956.

9M. M. Steiner, M. D. "Clínice Endocrinológica Pediátrica." Salvat Ed. (c) 1973, pp. 189.

${ }^{10}$ Grooks ans Share, L. "On the Question of Protein Binding and the Diffusibility and Ciredating Antidiuretic Hormone in the Dog." Endoerinology 78: 779, 1966.

11 Orloff and Handler, J. S. "The Similarity of Effects of Yasopressin Adenosin $3^{\prime} 5^{\circ}$ - Phosphate (Cyclic - Amp) and Theo philline on the Toad Bladder." Journal of Clinical Investigation 41: 702,1962 .

12 Gramtham y Bung, M. D. "Effects of Vasopressin and Cyclic Amp on Permeabjlity and Isolated Collecting Tubules." Arr. Jour. of Phis. 211: 255, 1966.

13 Handler et al. "The Effects of Vagupressin and the Theopbilline on the Concentration of Adenosin Phosphale in the Urinary Bladder of the Toad." Journal of Biological Chemistry 240: 4524, 1965.

14 Dotusa, T. P. R; W. Walter; H. Sands; t. L. Schwartz, and O. Hechter. 1972. "Rule of Cyclic Amp in the Action of Neuro- 
hypophyseal Hormones un the Kidney ". Adv. Cyclic Nucleotide. Res. 1: 121-135.

15 Brown, E., Clark, et ois. "The Stimulation of Adenosin 3'5" Monophosphate Production by Antidiuretic Factors". Journal of Biological Chemistry 236: 852.

${ }^{16}$ Nornan Bell et als. Demonstration of a Defect in the Formation of Adenusine 3'5' Monophosphate in Vampreysin-Registaut Diabetes Insipidus".

${ }^{17}$ Takahashi et als. "Effects of Yasupressin ard Water: Larad on I rinay Adenosine -3'-5' Monophogphate", Lancet 2: 967. 1966.

18 Orloff and itandler. "The Rule of Adenosin 3-5' Phosphale in the Action of Antidiurelic Hornone". Am. Jour. of Med. 42: 757768,1967 .

${ }^{19}$ Chandhtur, T. K., Winer, N. Effect of Chlorpropamide on Renal Phosphudiesterase (Absirac1) J. Lab. Chem. Med. 76: 863. 1970.

21) Thonas Dorsa. Cellular Action of Antidiuretic Hormone in Nephrogenic Diabetes Inyjpidus. Muyo Clinic Prus. Mar. 74. Fol. 49: 189-199.

${ }^{21}$ Granthum, J; Burg, M. B. (1966). E[fects of Vasopressin and Cyclic Amp on Permeability of Isolated Collecting Tubules. Am. Jour. P'hisjology. 211: 25.5.

22 Grantham, J.J. Vasupressin: Effects on Deformability of Urinanary surface of Collecting Duct Cells. Science 168: 1093-1095. 1970.
23 Thomas Douss and Heinz Volling. Ceilular Acton of Antidiuretic Hormune in . Hice with Inherited Vasopressin-Resistant Urinary Concentrating Defects". The Journad of Clinical Invesligation Vol. 54, Sept. 74: 753-762.

24 R. Monn et af, "Basal and Hormune Induced Urinary Cyclic Amp in Children witl Renal Disneders". Acila Pediatr. Scund. 65: $739,1976$.

25 Richord Schreister et als. "Langenital Nephrogenic biabetes Insipidus in a Baby Girls". Atchives of Disease in Chilidhord, 53: 906-915, 1978.

${ }^{26}$ Zinunernan and Oreille, C. Green. "Nephrogenic Diabetes Insipidus Type II: Defect Distal to the Adenilciclasa Step". Ped. Research. 4: 381, 1975.

2? Hobert Wien Bensel et al. "Prugressiye Hydronephrosis, Hydroureter and Dilatation of the Bladder in Sibtings with Congenital Ciephrogenie: Disbetes lnsipidus". The Joumal of Perliatrics. Yol. 77, No. 3, pp. 439-443.

${ }^{28}$ Marlyn, $C$.; Schottand, et ols. The Lffects of Chlorothiazide in Nephrogenic Diabetes Insipidus". Pediatries, May 1963, pp. 741-753.

${ }^{29}$ D. L. Duties and G. M. Wilscin. "Dinretics: Mechanism of Action and Clinical Aplication". Irugs. 9 (3i) 128-226, 1975.

${ }^{30}$ Comanicocion al Carso lusternucionat de Nefrotogio. Abrit 79. Hospital J. M. Ferrari. Chile. Dr. Eduardo Garm. 